\title{
Improving color quality and luminous flux of white LED utilizing triple-layer remote phosphor structure
}

\author{
Nguyen Thi Phuong Loan', Nguyen Doan Quoc Anh' \\ ${ }^{1}$ Faculty of Fundamental 2, Posts and Telecommunications Institute of Technology, Vietnam \\ ${ }^{2}$ Power System Optimization Research Group, Faculty of Electrical and Electronics Engineering, \\ Ton Duc Thang University, Vietnam
}

\begin{tabular}{l} 
Article Info \\
\hline Article history: \\
Received Aug 30, 2019 \\
Revised Apr 27, 2020 \\
Accepted May 8, 2020 \\
\hline
\end{tabular}

\section{Keywords:}

Color rendering index

Lambert-Beer law

Luminous efficacy

White LED

\begin{abstract}
In this manuscript, we presented a research that enhance the performance of WLED using the multi-phosphor configuration. The phosphor layers in the research are separated from each other to achieved better luminous efficiency, however, it makes controlling color light quality more complex. Another issue is finding out the whether two layers of phosphor or three layers of phosphor is better in improving color quality. The research addressed this issue by analyzing the optical aspects of the respective WLEDs that employ these structure. The studied aspects are quality indicators such as luminous efficacy (LE), and color uniformity, color rendering index (CRI), color quality scale (CQS). The results of the experiments in this research, which come from the employment of WLEDs with 2 color temperatures $5600 \mathrm{~K}$ and $8500 \mathrm{~K}$, suggest that WLED with three phosphor layers is better in CRI, CQS, LE. This type of phosphor structure also limits the color deviation significantly, thus, improves the color uniformity. This results is verifies with Mie theory, therefore, can be applied as reference or guideline for production of better WLEDs.
\end{abstract}

Copyright (c) 2020 Institute of Advanced Engineering and Science. All rights reserved.

\section{Corresponding Author:}

Nguyen Doan Quoc Anh,

Power System Optimization Research Group,

Faculty of Electrical and Electronics Engineering,

Ton Duc Thang University,

No. 19 Nguyen Huu Tho Street, Tan Phong Ward, District 7, Ho Chi Minh City, Vietnam.

Email: nguyendoanquocanh@tdtu.edu.vn

\section{INTRODUCTION}

Phosphor-converted white light-emitting diodes (WLEDs) are a promising light source because of their small size, high energy efficiency, low cost, and color stability [1-4]. WLEDs apply the principle of complementary colors: Blue light from a blue chip is combined with yellow light from phosphor [5]. WLEDs have the potential to be used in solid-state lighting, but their luminous efficiency must be enhanced [6, 7]. Generally, freely dispersed coating is the most common method used to fabricate white light. In this process, transparent encapsulated resin is combined with phosphor powder and is dispersed on the phosphor package. Although this approach allows the thickness of the phosphor layer to be controlled easily and reduces much of the cost, it does not produce high-quality WLEDs [8-10]. Therefore, the conformal coating method can be used as an alternative. This method distributes colors uniformly, resulting in angular homogeneity of correlated color temperature (CCT) [11], however, the luminous efficiency of WLED that has a conformal phosphor structure is reduced due to backscattering effect. To lessen the effect of backscattering and strengthen the luminous efficiency, previous studies have demonstrated the concept of remote phosphor structure by separating the chip and the phosphor layer [12, 13]. Moreover, the enhanced light extraction internal reflection structure that can increase extraction efficiency by using a polymer hemispherical shell lens with interior phosphor coating is also introduced [14]. Lastly, an air-gap embedded structure can 
enhance luminous efficiency by reflecting downward light [15]. These results show that the lumen output of WLEDs can benefit from remote phosphor configuration. While luminous efficacy (LE) is an important aspect to assess the quality of WLEDs, other optical properties including color rendering index (CRI), color quality scale (CQS), and color uniformity are also highly essential. Although there are many studies researching for luminous flux developments, documents regarding other optical properties are lacking. For example, there is study about the influences of distance between phosphor layers in flat dual-remote phosphor (FDRP) structure and concave dual-remote phosphor structure (CDRP) on luminous flux that confirms FDRP benefits the luminous efficiency and proposes an optimal distance option. The distances between phosphor layers in triple-remote phosphor structure are also studied to boost the light output. Moreover, the study of SrBaSiO4: $\mathrm{Eu}^{2+}$ particles in controlling WLEDs green light output with conformal phosphor packages was also accounted for luminous flux and color homogeneity development. As can be seen, luminous efficiency received abundant attention while other matters such as the impacts of layers distance in triple-remote phosphor structure and phosphor concentration on other optical properties are being neglected. Therefore, the remote phosphor configuration is applied in multi-phosphor WLEDs with the results being compared to find the optimal strategy to improve the optical quality of WLEDs. This is the first time the optical properties from these two remote-phosphor configurations, such as color rendering index (CRI), color quality scale (CQS), and color uniformity, are being measured and compared. In the dual-layer phosphor configuration, yellow phosphor is lower, upper is red phosphor or green phosphor [16-18]. In the triple-layer phosphor configuration, yellow phosphor is lower, red phosphor is upper, and green phosphor is middle. The phosphor package essential factor in deciding the luminous efficiency, however, the concentration of phosphor holds equal importance because the higher phosphor concentration the more light is loss from re-absorption. This effect can be observed from WLEDs with low CCT that have insufficient luminous efficiency $[19,20]$. An obvious solution is to boost the emitted light from the light sources while limiting damage to extracted light. The framework of the manuscript is divided to three main parts: the first part is the presentation of the WLED model being used in the experiments and its parameters, second part are the mathematical equation for the computation of the optical properties, and the third part is the illustration and comparison of calculated results in graphs. The methods to improve optical properties of WLEDs might be available, however, not all are updated and the lack of condensation can confuse the manufacturers and make choosing phosphor structure extremely difficult for the manufacturers. Hence, this research proposes the best selection for manufacturers to improve WLEDs quality. The method for improvement are specialized for different cases that allow manufacturers to choose the one that can obtain the optical properties of their desired WLED.

\section{RESEARCH METHOD}

The ideas of this research is to increase the luminous flux with $\mathrm{SrBaSiO}_{4}: \mathrm{Eu}^{2+}$ by promoting emitted green light and enhance CRI, CQS by supplying WLEDs with more material for red light from $\mathrm{Sr}_{\mathrm{w}} \mathrm{F}_{\mathrm{x}} \mathrm{B}_{\mathrm{y}} \mathrm{O}_{\mathrm{z}}: \mathrm{Eu}^{2+}, \mathrm{Sm}^{2+}$. In this research, WLEDs have 9 LED chips inside, as Figure 1(a) suggests. The wattage of blue chip is $1.16 \mathrm{~W}$ at the peak wavelength of $453 \mathrm{~nm}$ for each individual. The technical parameters details of LED configuration can be seen from Figure 1(b). Figure 1(c) demonstrates single-layer remote phosphor (Y) structure with yellow phosphor YAG: $\mathrm{Ce}^{3+}$ as the only phosphor material placed on the surface of LEDs chips. Figure 1(d) demonstrates the YG structure with red phosphor $\mathrm{Sr}_{\mathrm{w}} \mathrm{F}_{\mathrm{x}} \mathrm{B}_{\mathrm{y}} \mathrm{O}_{\mathrm{z}}$ : $\mathrm{Eu}^{2+}, \mathrm{Sm}^{2+}$ above yellow phosphor YAG: $\mathrm{Ce}^{3+}$ while Figure 1(e) demonstrates YG structure that has green phosphor layer $\mathrm{SrBaSiO}_{4}: \mathrm{Eu}^{2+}$ and yellow phosphor instead. The triple-layer remote structure has phosphor layer $\mathrm{SrBaSiO}_{4}: \mathrm{Eu}^{2+}$ which is between 2 phosphor layers illustrated in Figure 1(f). This remote phosphor layers has a range of $0.05 \mathrm{~nm}$. The YAG:Ce ${ }^{3+}$ concentration must change with the yellow or red phosphor concentration to maintain the average correlated color temperature (ACCTs), the content of Figure 2 will illustrate this event in details. The YAG:Ce ${ }^{3+}$ concentration in each phosphor structure at any distinct ACCT is also different, which leads to scattering properties diversity in LED and different optical properties values.

It is easy to realize in Figure 2, yellow-emitting YAG:Ce ${ }^{3+}$ phosphor concentration attains the maximum value at $\mathrm{Y}$ structure and minimum value at YRG structure in all ACCTs. At remote phosphor structures within the same ACCT, the higher YAG:Ce ${ }^{3+}$ concentration, the higher backscattering ability, result in reducing luminous flux emission. Furthermore, if the YAG: $\mathrm{Ce}^{3+}$ concentration is high, there will be the unbalance among 3 basic colors which create yellow, red, and green light that cause a decrease in the color quality of WLEDs. Therefore, to improve the luminous flux and chromatic performance of WLEDs, it is necessary to reduce the backscattering and maintain the balance among 3 basic colors yellow, red, and green. Moreover, by increasing the red light component, we can control the color rendering index. Additionally, the chromatic homogeneity and luminescence can be controlled by the green light component.

Improving color quality and luminous flux of white LED utilizing triple-layer... (Nguyen Thi Phuong Loan) 
From the above analysis, is the triple-layer phosphor structure the most advantageous in controlling the optical properties? To answer this question, the researchers continue to give important information of remote phosphor structures and emission spectrum, which results are presented in Figure 3.

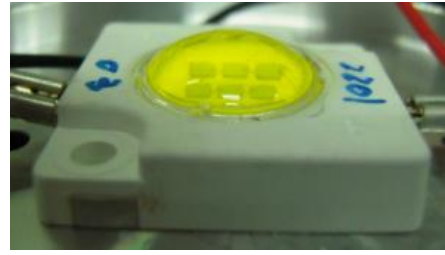

(a)

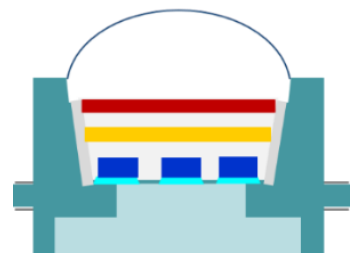

(d)

\author{
Lead frame: $4.7 \mathrm{~mm}$ Jentech Size-S \\ LED chip: $\mathrm{V} 45 \mathrm{H}$ \\ Die attach: Sumitomo 1295SA \\ Gold Wire: 1.0 mil \\ Phosphor: ITC NYAG4_EL
}

(b)

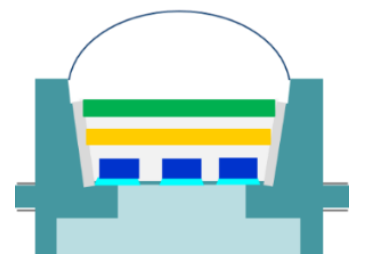

(e)

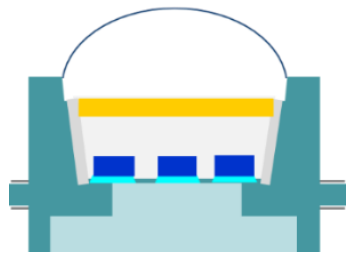

(c)

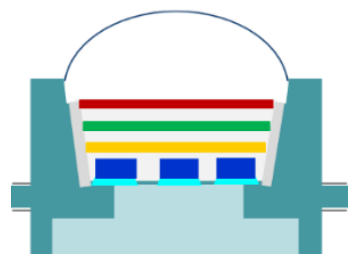

(f)

Figure 1. Illustration of multi-layer phosphor structures of white LEDs:

(a) the MCW-LEDs and (b) technical parameters; (c) single-layer phosphor, dual-layer remote phosphor with (d) YR and (e) YG, and (f) triple-layer phosphor

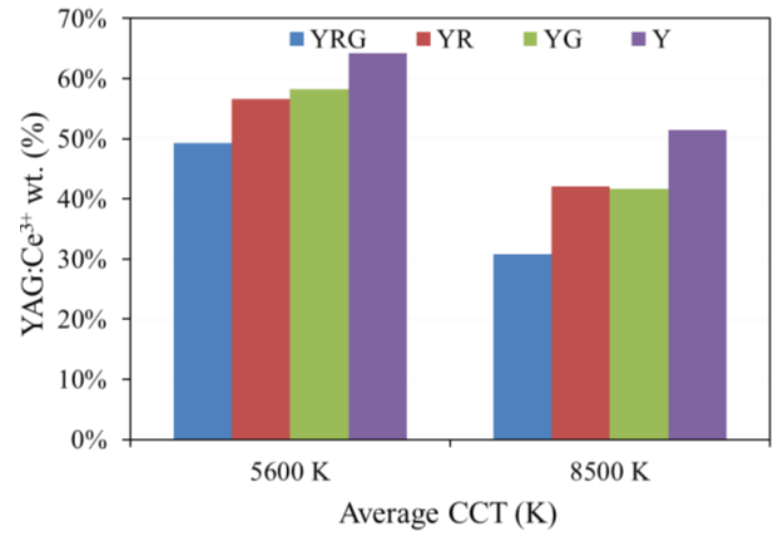

Figure 2. Yellow-emitting YAG:Ce3+ phosphor concentration corresponds to each remote phosphor structure in each ACCT
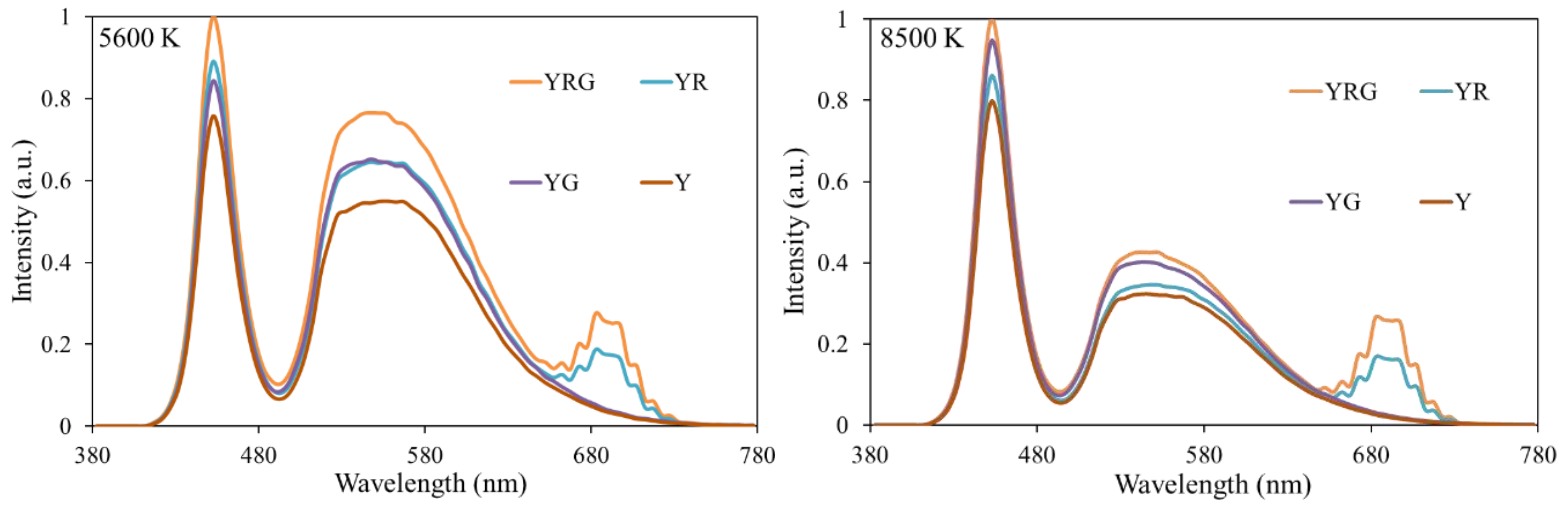

Figure 3. Emission spectra of phosphor configurations 
There is an obvious distinction in the emission spectrum in remote phosphor structures. When comparing with other three remote phosphor structures at 2 ACCTs, the Y emitter spectrum has the lowest intensity, which means the luminous flux produced by the Y structure is the smallest. In the other hand, the YRG structure has the highest spectral intensity in the wavelength range from $380 \mathrm{~nm}$ to $780 \mathrm{~nm}$. In the wavelength range from $400 \mathrm{~nm}$ to $500 \mathrm{~nm}$, the spectral intensity of the YG structure is higher than of the YR structure, so the luminous flux of the YG structure may be greater than of the YR structure. However, the spectral intensity of the YR structure from $650 \mathrm{~nm}$ to $750 \mathrm{~nm}$ is higher than of the YG structure, which can make YR have a better color rendering index than YG does. To confirm the above analysis, we need to consider the result in section 3 .

\section{RESULTS AND ANALYSIS}

Figure 4 shows the comparison of CRI between remote phosphor structures. Obviously, the YR structure can achieve the highest CRI at any ACCT. This is the crucial result for improving the CRI of the remote phosphor structures. In controlling CRI, there is a challenge to control a high ACCT (greater than $5600 \mathrm{~K})$, but the YR layer can perform this. The YR structure can bring benefit in CRI due to the red light component added from the red phosphor layer $\mathrm{Sr}_{\mathrm{w}} \mathrm{F}_{\mathrm{x}} \mathrm{B}_{\mathrm{y}} \mathrm{O}_{\mathrm{z}}: \mathrm{Eu}^{2+}, \mathrm{Sm}^{2+}$. Regarding the CRI value that can be achieved, the YRG stands at the second while the YG structure stands at the last. Therefore, if the goal is the CRI, we can choose the YR structure in WLEDs mass production. Nevertheless, CRI is just one of the indicators to evaluate the color quality. In the recent years, CQS has become the target of many researches. CQS is the combination of 3 elements: CRI, visual preference, and color coordinate. With these three components, CQS has become the large target and the most vital indicator for evaluating color quality. In this study, the comparison between CQS of the remote phosphor structures is shown in Figure 5.

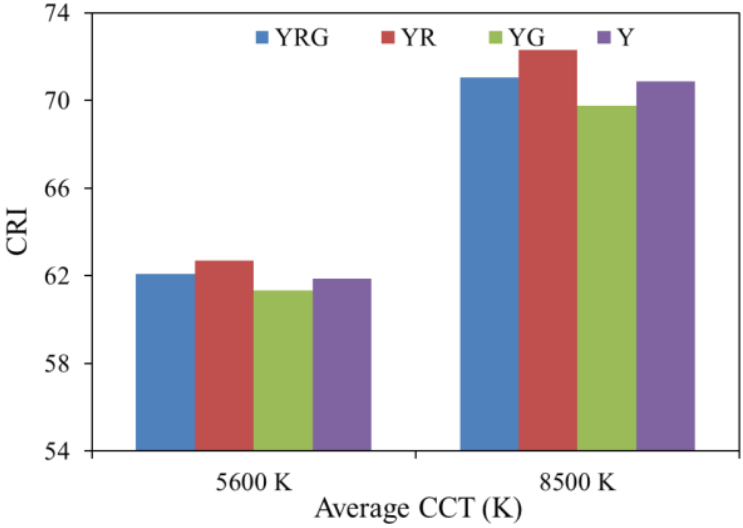

Figure 4. Color rendering indexes of phosphor configurations corresponding to ACCTs

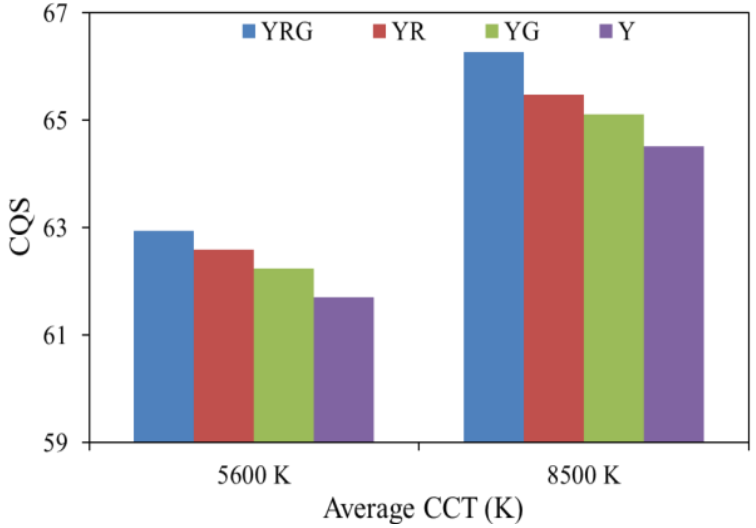

Figure 5. Color quality scale of phosphor configurations corresponding to ACCTs

If the YR reaches the highest CRI, the YRG reaches the highest CQS, which can be explained by the stability of the three basic colors-yellow, red, and green. The higher the CQS value is, the higher the color quality. The Y structure produces the lowest CQS. In general, the Y structure has luminous flux advantage, but has difficulty in controlling color quality without the addition of the red light and the green light components. Although the Y structure has disadvantage in color quality, it brings benefits to production. Its production is easier than other structures' and the cost may be reduced.

From the above analysis, the YRG structure should be chosen. However, if the color quality is better, will the luminous flux be affected in Figure 6? Can we confirm that if the producers' goal is color quality, will it be affected? To answer this problem, the researchers conducted the comparison of the luminous flux emitted from the single-layer and the dual-layer. The equation to compute the transmitted blue light and converted yellow light in the dual-layer phosphor structure, which can lead to a vast enhancement of LED quality, will be present and illustrate in this part. The equations for converted yellow light and the transmitted blue light for remote phosphor WLED with one layer of phosphor are shown below, the layer thickness is represented by $2 h$ :

$$
P B_{1}=P B_{0} \times e^{-2 \alpha_{B 1} h}
$$




$$
P Y_{1}=\frac{1}{2} \frac{\beta_{1} \times P B_{0}}{\alpha_{B 1}-\alpha_{Y 1}}\left(e^{-2 \alpha_{Y 1} h}-e^{-2 \alpha_{B 1} h}\right)
$$

The converted yellow light and the transmitted blue light for dual-layer remote phosphor WLEDs with the thickness of the layers depicted as $\mathrm{h}$ are defined as:

$$
\begin{aligned}
& P B_{2}=P B_{0} \times e^{-2 \alpha_{B 2} h} \\
& P Y_{2}=\frac{1}{2} \frac{\beta_{2} \times P B_{0}}{\alpha_{B 2}-\alpha_{Y 2}}\left(e^{-2 \alpha_{Y 2} h}-e^{-2 \alpha_{B 2} h}\right)
\end{aligned}
$$

$\mathrm{h}$ is the thickness of each phosphor layer. The subscripts "l" and "2" are used as the description of the single-layer and double-layer remote phosphor package. While $\beta$ presents for the conversion coefficient for blue light converted to yellow light, $\gamma$ is the reflection coefficient of the yellow light. $P B_{0} . \alpha B ; \alpha Y$, which are the parameters describing the fractions of the energy lost for blue and yellow light during their propagation in the phosphor layer, indicates the intensities of blue light $(P B)$ and yellow light $(P Y)$ from blue LED. The lighting efficiency of pc-LEDs with the double-layer phosphor structure can enhance remarkably comparing to the one with the single-layer structure:

$$
\frac{\left(P B_{2}+P Y_{2}\right)-\left(P B_{1}+P Y_{1}\right)}{P B_{1}+P Y_{1}}>0
$$

By using the Mie theory [21, 22], the scattering of phosphor particles was analyzed. Additionally, in the following expression by using the Mie theory, the scattering cross section Cscafor spherical particles can be calculated. The transmitted light power can be computed by the Lambert-Beer law [23-25]:

$$
I=I_{0} \exp \left(-\mu_{\text {ext }} L\right)
$$

In this formula, $I_{0}$ is the incident light power, $L$ is the phosphor layer thickness (mm), and $\mu$ ext is the extinction coefficient, which can be expressed as: $\mu_{e x t}=N_{r} . C_{e x t} . N_{r}$ is the number density distribution of particles $\left(\mathrm{mm}^{-3}\right)$ and $C_{\text {ext }}\left(\mathrm{mm}^{2}\right)$ is the extinction cross-section of phosphor particles.

The 5 th expression proved that the usage of multiple phosphor layers has greater advantage for luminous flux than the one of single layer. The result was clearly proved in Figure 6, the Y structure reaches the lowest luminous flux, compares to the other four structures in every ACCTs. In contrast, the highest luminous flux was achieved by the YRG structure. There is no doubt that YRG can obtain the highest luminous flux when the color quality YRG is the best. The second highest luminous flux was achieved by the YG structure due to the green phosphor layer $\mathrm{SrBaSiO}_{4}: \mathrm{Eu}^{2+}$. The green phosphor layer $\mathrm{SrBaSiO}_{4}: \mathrm{Eu}^{2+}$ help to increase the green light component and the spectrum in the wavelength range from $500 \mathrm{~nm}$ to $600 \mathrm{~nm}$. Obviously, in this wavelength range, the intensity of YGR is greater than of YG and Y. Because of the concentration of the smallest phosphor YAG: $\mathrm{Ce}^{3+}$ in the YRG structure, ACCT can be maintained. The YRG structure reduces the amount of scattered light after YAG:Ce ${ }^{3+}$ concentration decreases. Blue light rays from LED chips are easily transmitted straight through the YAG:Ce ${ }^{3+}$ layer through other layers. In other words, the YRG structure helps blue light energy from the LED chip to convert efficiently. Therefore, the YRG spectral intensity is the highest compared to other remote phosphor structures in the white light wavelength range. And accordingly, the luminous flux of the YRG structure is also the highest.

Thus, the YRG structure can be selected by the superior optical properties of WLEDs including CQS and LE. However, it is not possible to ignore color copper when it comes to color quality factor. There are many methods to improve copper color, including methods of using advanced scattering particles such as $\mathrm{SiO}_{2}, \mathrm{CaCO}_{3}, \ldots$ or using conformal phosphor configuration. Although improved color uniformity, luminous flux can be significantly reduced if the above two methods are applied. The use of green $\mathrm{SrBaSiO}_{4}: \mathrm{Eu}^{2+}$ phosphor and red $\mathrm{Sr}_{\mathrm{w}} \mathrm{F}_{\mathrm{x}} \mathrm{B}_{\mathrm{y}} \mathrm{O}_{\mathrm{z}}: \mathrm{Eu}^{2+}, \mathrm{Sm}^{2+}$ phosphor has both increased scattering properties but also added a green or red light component inside WLEDs, to produce more white light. The use of a remote phosphor structure enhances the luminous flux emitted by reducing reflections back to the LED chip. However, it is necessary to control the suitable phosphor layer concentration to achieve the highest transfer energy. This can be proved by Lambert-Beer law's law in Expression 6.

Figure 7 shows the comparison of color differences between structures. The smaller the color deviation, the higher the color uniformity. Here, it is easy to see that the color difference of the YRG is the smallest. This can be explained by scattering inside the LED package before forming white light. 
The more phosphor layers there are, the more scattering events. And the result is increased color uniformity of WLEDs. Of course, when there are many scattering events, a decrease in luminous flux can occur. However, this reduction is negligible compared to the benefit obtained when the following scattering is reduced. Therefore, the YRG structure achieves the best color uniformity with the highest luminous flux. In contrast, the highest color deviation is expressed in the Y structure of all ACCTs.

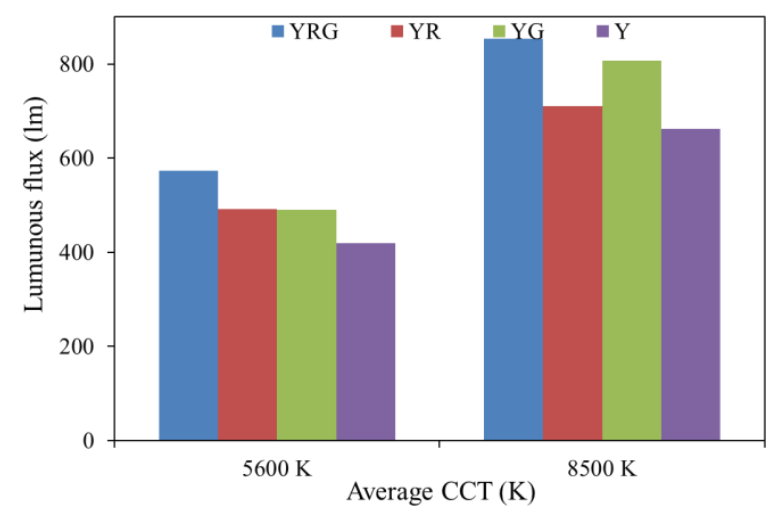

Figure 6. Luminous flux of phosphor configurations corresponding to ACCTs

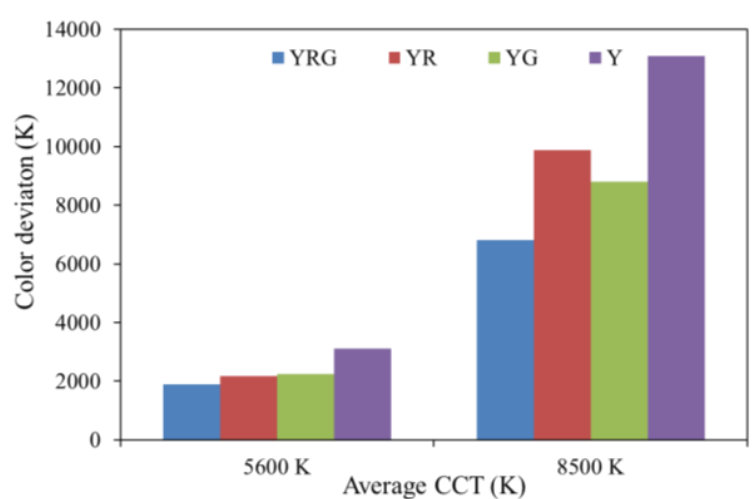

Figure 7. Correlated color temperature deviation (D-CCT) of remote phosphor configurations corresponding to ACCTs

\section{CONCLUSION}

This paper compares the optical performance of the four structures Y, YG, YR and YRG in the five ACCTs. Green $\mathrm{SrBaSiO}_{4}: \mathrm{Eu}^{2+}$ phosphor and red $\mathrm{Sr}_{\mathrm{w}} \mathrm{F}_{\mathrm{x}} \mathrm{B}_{\mathrm{y}} \mathrm{O}_{\mathrm{z}}: \mathrm{Eu}^{2+}, \mathrm{Sm}^{2+}$ phosphor is used in the simulation process. In addition, the study results were verified by the Mie theory and the Lambert-Beer law. Accordingly, adding green $\mathrm{SrBaSiO}_{4}: \mathrm{Eu}^{2+}$ phosphor adds green light to improve copper color and luminous flux. Therefore, the YG structure achieves better optical flux and color uniformity than the YR structure. CRI and CQS can be improved when increasing the red light component through the red $\mathrm{Sr}_{\mathrm{w}} \mathrm{F}_{\mathrm{x}} \mathrm{B}_{\mathrm{y}} \mathrm{O}_{\mathrm{z}}$ : $\mathrm{Eu}^{2+}, \mathrm{Sm}^{2+}$ phosphor. As a result, YR structure achieves higher CRI and CQS than YG. The color quality depends on the balance between the three primary colors yellow, green and red. The YRG structure, which possesses 3 phosphor layers each with a distinct shade from the three primary colors, can easily control the balance between colors and achieves the highest color quality out of all structures. In addition, the decrease in backscattering of YRG helps the luminous flux of this configuration significantly increase. The evidence is that the luminous flux achieved in the YRG structure is the also higher than other structures. The CRI in YRG structure might be slightly lower than YR structure, however, YRG structure compensate with higher CQS, which is more valuable quality indicator. Together with high chromatic stability, which can be inferred from the lowest correlated color temperature deviation (D-CCT), YRG structure is certainly the optimal structure for the fabrication of WLEDs with best optical quality. The wide coverage of this research provide a comprehensive view on the influences of each phosphor structure on WLEDs final performance. These information are valuable documents for reference regarding the improvement of WLEDs. The results of this study suggest an effective configuration for manufacturers to apply in WLEDs quality improvement and can be viewed as reference for future research.

\section{REFERENCES}

[1] P. X. Le, et al., "The Impacts of Distance Between Phosphor Layers on Optical Properties of Triple-Layer Phosphor Structure," Advances in Electrical and Electronic Engineering, vol. 17, no. 1, pp. 81-86, 2019.

[2] T. T. Trang, et al., "Excellent luminous flux of WLEDs with flat dual-layer remote phosphor geometry," TELKOMNIKA Telecommunication Computing Electronics and Control, vol. 17, no. 5, pp. 2527-2534, 2019.

[3] W. L. Zhang, et al., "Spectral optimization of color temperature tunable white LEDs based on perovskite quantum dots for ultrahigh color rendition," Optical Materials Express, vol. 7, issue 9, pp. 3065-3076, 2017.

[4] Q. R. Yan, et al., "Improved color rendering of phosphor-converted white light-emitting diodes with dual-blue active layers and n-type AlGaN layer," Optics Letters, vol. 37, pp. 1556-1558, 2012.

[5] S. Jost, et al., "CIE 2017 color fidelity index Rf: a better index to predict perceived color difference?," Journal of the Optical Society of America A, vol. 35, issue 4, pp. B202-B213, 2018. 
[6] S. P. Ying, et al., "Color design model of high color rendering index white-light LED module," Applied Optics, vol. 56, issue 14, pp. 4045-4051, 2017.

[7] L. Wang, et al., "Enriching red emission of $\mathrm{Y}_{3} \mathrm{Al}_{5} \mathrm{O}_{12}$ : $\mathrm{Ce}^{3+}$ by codoping $\mathrm{Pr}^{3+}$ and $\mathrm{Cr}^{3+}$ for improving color rendering of white LEDs," Optics Express, vol. 18, issue 24, pp. 25177-25182, 2010.

[8] A. David, et al., "Whitehead L.Development of the IES method for evaluating the color rendition of light sources," Optics Express, vol. 23, issue 12, pp. 15888-15906, 2015.

[9] H. K. Lee, et al., "Color-tunable organic light-emitting diodes with vertically stacked blue, green, and red colors for lighting and display applications," Optics Express, vol. 26, issue 14, pp. 18351-18361, 2018.

[10] Y. Chen, et al., "Comments on "Maximum White Luminous Efficacy of Radiation Versus Color Rendering Index and Color Temperature: Exact Results and a Useful Analytic Expression,” Journal of Display Technology, vol. 9, pp. 859-860, 2013.

[11] Y. Yu, et al., "Improving the color-rendering index of a tandem warm white organic light-emitting device by employing a simple fabrication process," Optics Letters, vol. 44, pp. 931-934, 2019.

[12] P. Zhong, et al., "Spectral optimization of the color temperature tunable white light-emitting diode (LED) cluster consisting of direct-emission blue and red LEDs and a diphosphor conversion LED," Optics Express, vol. 20, issue 55, pp. A684-A693, 2012.

[13] C. F. Lai, et al., "Saving Phosphor by $150 \%$ and Producing High Color-Rendering Index Candlelight LEDs Containing Composite Photonic Crystals," Journal of Lightwave Technology. vol. 32, no. 10, pp. 1930-1935, 2014.

[14] K. A. G. Smet and P. Hanselaer, "Impact of cross-regional differences on color rendition evaluation of white light sources," Optics Express, vol. 23, issue 23, pp. 30216-30226, 2015.

[15] J. H. Oh, et al., "Excellent color rendering indexes of multi-package white LEDs," Optics Express, vol. 20, issue 18, pp. 20276-20285, 2012.

[16] T. T. Trang, et al., "Improving luminous flux and color homogeneity of dual-layer phosphor sctructure," TELKOMNIKA Telecommunication Computing Electronics and Control, vol. 17, no. 5, pp. 2643-2649, 2019.

[17] L. Y. Chen, et al., "Chromaticity tailorable glass-based phosphor-converted white light-emitting diodes with high color rendering index," Optics Express, vol. 23, issue 15, pp. A1024-A1029, 2015.

[18] L. L. A. Price, et al., "Entropy, color, and color rendering," Journal of the Optical Society of America, vol. 29, issue 12, pp. 2557-2565, 2012.

[19] J. M. Quintero, et al., "Color rendering map: a graphical metric for assessment of illumination," Optics Express, vol. 20, issue 5, pp. 4939-4956, 2012.

[20] J. M. M. Linhares, et al., "Color rendering of art paintings under CIE illuminants for normal and color deficient observers," Journal of the Optical Society of America, vol. 26, issue 7, pp. 1668-1677, 2009.

[21] K. C. L. Lin, et al., "Approach for optimization of the color rendering index of light mixtures," Journal of the Optical Society of America, vol. 27, issue 7, pp. 1510-1520, 2010.

[22] G. Cheng, et al., "Pure white hybrid light-emitting device with color rendering index higher than 90," Optics Letters, vol. 35, issue 5, pp. 616-618, 2010.

[23] R. Mirhosseini, et al., "Improved color rendering and luminous efficacy in phosphor-converted white light-emitting diodes by use of dual-blue emitting active regions," Optics Express, vol. 17, issue 13, pp. 10806-10813, 2009.

[24] S. Nizamoglu, "Warm-white light-emitting diodes integrated with colloidal quantum dots for high luminous efficacy and color rendering: reply to comment," Optics Letters, vol. 36, issue 15, pp. 2852-2852, 2011.

[25] W. A. Thornton, "Luminosity and Color-Rendering Capability of White Light," Journal of the Optical Society of America, vol. 61, issue 9, pp. 1155-1163, 1971. 